\title{
Systemic corticosteroids for management of 'long-COVID': an evaluation after 3 months of treatment
}

\author{
Nitin Goel' ${ }^{1}$, Nitesh Goyal ${ }^{1}$, Ravishankar Nagaraja ${ }^{2}$, Raj Kumar ${ }^{1}$ \\ ${ }^{1}$ Department of Pulmonary Medicine; ${ }^{2}$ Department of Biostatistics, Vallabhbhai Patel Chest Institute, University of Delhi, \\ India
}

\begin{abstract}
Some patients even 4 weeks after Coronavirus Disease 2019 (COVID-19) remain to be symptomatic and are known as "longCOVID". In the present study we performed the follow up evaluation at 3 months of long-COVID patients, after treatment with systemic steroids. During the study duration, out of the 4,542 patients managed in the outpatient department of the particular unit, there were 49 patients of long-COVID. The patients having abnormal computed tomography (CT) along with resting hypoxia or exertional desaturation were treated with systemic steroid (deflazacort) in tapering doses for 8-10 weeks. We retrospectively
\end{abstract}

Correspondence: Nitin Goel, M.D. Assistant Professor, Department of Pulmonary Medicine, Room No. 2, Viswanathan Chest Hospital, Vallabhbhai Patel Chest Institute, University of Delhi, Delhi 110007, India.

Tel. +91.9717317899 / +91.011.27667667 Extn 185 .

E mail: drnitingoel@gmail.com

Key words: COVID-19; corticosteroids; SARS-CoV-2; hypoxia, breathlessness.

Contributions: NGoe, conception and design, analysis and interpretation of data, drafting the article; NG, analysis and interpretation of data, drafting the article; $\mathrm{RN}$, analysis and interpretation of data; $\mathrm{RK}$, analysis and interpretation, revising it critically. All authors have approved the final version and agreed to be accountable for all aspects of the work.

Conflict of interest: The authors declare that they have no competing interests, and all authors confirm accuracy.

Ethics approval: This retrospective analysis was conducted after due approval from the Institutional Ethics Committee.

Informed consent: Obtained from participants. The manuscript does not contain any individual person's data in any form.

Received for publication: 16 June 2021.

Accepted for publication: 28 September 2021.

${ }^{\circ}$ Copyright: the Author(s), 2021

Licensee PAGEPress, Italy

Monaldi Archives for Chest Disease 2022; 92:1981

doi: 10.4081/monaldi.2021.1981

This article is distributed under the terms of the Creative Commons Attribution Noncommercial License (by-nc 4.0) which permits any noncommercial use, distribution, and reproduction in any medium, provided the original author(s) and source are credited. analysed the clinical and radiological findings of these patients at first presentation and at about 3 months of follow up visit. On follow up, all the 49 long-COVID patients showed improvement. The occurrence of breathlessness decreased from $91.83 \%$ to $44.89 \%(\mathrm{p}<0.001)$ and cough from $77.55 \%$ to $8.16 \%(\mathrm{p}<0.001)$. Twenty-four patients were prescribed systemic steroids. Out of these, nearly $58 \%$ patients had MMRC grade 4 breathlessness, which decreased to $\leq 2 \mathrm{MMRC}$ in about $86 \%$ of these patients. MMRC grade (median) decreased from 3 to 1 ( $\mathrm{p}<0.001)$. Majority of patients who were tachypnoeic and hypoxic at rest $(n=7)$ showed improvement (71\%), post-treatment with corticosteroids. Occurrence of normal chest X-ray increased from $12 \%$ to $71 \%$ $(\mathrm{p}<0.001)$. All these patients had abnormal CT thorax initially, and post-treatment $25 \%$ had normal CT thorax. Hence, we conclude that systemic steroids are helpful in hastening recovery of select subset of long-COVID patients. Simultaneously, we should be cautious of immunosuppressive effects of steroids like tuberculosis reactivation, especially in tuberculosis endemic countries. These findings have therapeutic implications and may serve as guidance for future approach to the management of 'longCOVID' with pulmonary sequalae.

\section{Introduction}

Corona virus disease (COVID-19) caused by severe acute respiratory syndrome coronavirus 2 (SARS-CoV-2) is known for high infectivity and mortality [1]. Early diagnosis and prompt treatment of COVID-19 have been the topmost priority of the healthcare systems in recent times [2,3]. Lot of literature and guidelines are now available to describe its symptoms and varied clinical presentations $[4,5]$. Newer studies have found out that patients continue to experience symptoms even after resolution of the acute COVID-19 infection. These symptoms can range from milder ones to serious organ specific manifestations [6,7]. Terms like 'post-COVID syndrome', 'long haul COVID' and 'post-COVID sequelae' have been used in the literature for such persistent signs and symptoms [8]. The National Institute for Health and Care Excellence (NICE) guideline has coined the term 'long-COVID' for symptoms persistent beyond 4 weeks from acute COVID-19 infection [9]. In few studies, it was found that fatigue and dyspnoea are the most common persistent symptoms [10,11]. In another study, 3 monthly follow up of post-COVID patients for 1 year was done and evidence of persistent physiological and radiological changes in the lungs was found even after 1 year [12]. In this study, we retrospectively evaluated the clinical and radiological outcomes of the longCOVID patients after 3 months of management. 


\section{Materials and Methods}

The study was conducted one of the units of the Department of Pulmonary Medicine at Viswanathan Chest Hospital, Vallabhbhai Patel Chest Institute, University of Delhi, India. Our hospital is a tertiary care centre for management of the respiratory diseases. The study is a retrospective analysis of the long-COVID patients' provided consultation in the OPD (outpatient department) at the particular unit from 17 June 2020 to 15 January 2021.

\section{Case definition}

Patient with history of diagnosis of COVID-19 by RT-PCR test or RAT (rapid antigen test) and had presented to us for evaluation in the OPD after 4 weeks of diagnosis of COVID-19, were recruited in the study.

\section{Long-COVID management protocol}

While evaluating long-COVID patients in our OPD, we perform routine blood investigations, sputum examination, six-minute walk test (6MWT), chest X-ray, HRCT (high resolution computed tomography) scan of the thorax and any other investigation as indicated:

1. Domiciliary oxygen support is prescribed to those patients who are hypoxic at rest, to target a $\mathrm{SpO}_{2}$ of $\geq 90 \%$.

2. Patients with abnormalities in HRCT thorax (reticulations or ground glass opacities or parenchymal bands) and any of the following indications are prescribed with systemic corticosteroids: i) hypoxia at rest - $\mathrm{SpO}_{2}<90 \%$; ii) significant desaturation ( $>4 \%$ fall in oxygen saturation) in six-minute walk test. We use steroids to manage these long-covid patients in corollary to treatment protocol of inflammatory ILDs (like sarcoidosis and hypersensitivity pneumonitis) [13]. Although these guidelines recommend corticosteroids at a dose of $0.5-1 \mathrm{mg} / \mathrm{kg}$ prednisolone and tapering over a period of weeks to months, we usually prescribe a lower dose of steroids. Steroid (prednisolone) is usually prescribed in dose of $0.25-0.5 \mathrm{mg} / \mathrm{kg}$ body weight initially, after ruling out any active infection. Deflazacort in equivalent doses is usually preferably used. The usual prescription pattern is to initiate with tablet deflazacort $36 \mathrm{mg}$ daily for 1 week followed by $30 \mathrm{mg}$ for 1 week, $18 \mathrm{mg}$ for 1 week, $12 \mathrm{mg}$ for 1 week, $6 \mathrm{mg}$ for 2 weeks and to stop after $6 \mathrm{mg}$ alternate day for 2 weeks. Doses are tapered in this manner gradually (on an average of over $8 \pm 2$ weeks) depending upon the clinical and radiological recovery.

3. Inhaled corticosteroids (budesonide 400-800 $\mu \mathrm{g}$ /day) with or without inhaled long-acting bronchodilator (formoterol 12-24 $\mu \mathrm{g} /$ day) is prescribed to those patients who complain of wheezing or have rhonchi on auscultation.

\section{Study protocol}

File records of these patients were retrieved for analysis. All the details both at the time of presentation and follow up were analysed. These included demographic details, clinical symptoms, physical examination findings, investigations-reports and treatment given for the long-COVID. Only those patients of long-COVID, with available file records of at least 12 weeks after the first presentation to the OPD of the study unit, were included in the study.

The present study was conducted after due approval from the Institutional Ethics Committee (IEC). The informed consent was obtained from the participants.

\section{Statistical analysis}

The collected data were entered in a Microsoft Excel spreadsheet. The data were imported and analysed in SPSS v22 statistical software. The variables pertaining to this study were mostly categorical, except age. Categorical variables were summarized using frequency and percentage in each of the categories. Mean and standard deviation was computed for the variable age. McNemar test was performed to compare the proportion of the events before and after the intervention; $\mathrm{p}$-value less than 0.05 was considered as statistically significant [14].

\section{Results}

About 4,542 patients were managed in the OPD of the specific unit of the Department of Pulmonary Medicine during the study duration. Amongst them, there were 49 patients of the long-COVID fulfilling the inclusion criteria and these were evaluated in the present study. The average duration of follow up evaluation (from the first presentation to the OPD) of these patients was 12-14 weeks. The baseline characteristics of these patients are summarised in Table 1 . Around $30 \%(\mathrm{n}=15)$ of the patients had mild disease and had had recovered in the home isolation only. Rest of the patients had history of moderate to severe COVID- 19 . Majority $(80 \% ; n=12)$ of the home isolated patients had evidence of abnormalities on chest radiography at presentation. The most common co-morbidity was diabetes mellitus $(n=17 ; 34.69 \%)$. Nearly $88 \%(n=15)$ of the diabetic patients required hospitalisation for the COVID-19 illness. Further, all the patients with diabetes mellitus had reticulations on chest radiography at presentation. Nearly $8 \%$ patients $(n=4)$ were found to have raised blood sugars for the first time after they developed COVID-19 infection. Allergic rhinitis ( $n=9 ; 18.36 \%)$ was the most common respiratory comorbidity. Most common symptoms at presentation were fatigue $(\mathrm{n}=46 ; 93.8 \%)$ followed by breathlessness $(n=45 ; 91.83 \%)$ and cough $(n=38,77.55 \%)$.

At the time of presentation, $11(22.45 \%)$ patients were anaemic, 9 (18.37\%) had leucocytosis, $5(10.21 \%)$ had thrombocytopenia and $4(8.16 \%)$ had thrombocytosis. Kidney function test were normal in all the patients. Liver enzymes were mildly deranged (less than 2 times upper normal limit) in $20(40.82 \%)$ patients. Specialist consultation was advised to all the patients with blood biochemistry abnormalities and at the time of follow up none of the patient had any derangement in these investigations.

There were 7 (14.28\%) patients with resting hypoxia. Arterial blood gas analysis was performed for these patients and it revealed mean $\mathrm{pH}$ of $7.43 \pm 0.024, \mathrm{pCO}_{2}$ of $36.14 \pm 2.53$ and $\mathrm{paO}_{2}$ of $51.28 \pm 2.54$. They were prescribed domiciliary oxygen therapy at 1-2 litres per min to maintain a $\mathrm{SpO}_{2}$ of ${ }^{3} 90 \%$.

The details of treatment prescribed for long-COVID is depicted in Table 1. About $49 \%(n=24)$ of the patients were prescribed oral corticosteroids in tapering doses for 6 to 8 weeks. Out of these, $75 \%(n=18)$ did not have any pre-existing respiratory illness. 
The follow up evaluation of these patients is depicted in Table 2. It was found that the prevalence of fatigue decreased from $93.87 \%$ to $51.02 \%(p<0.001)$, breathlessness from $91.83 \%$ to $44.89 \%(\mathrm{p}<0.001)$ and cough from $77.55 \%$ to $8.16 \%(\mathrm{p}<0.001)$.

On chest radiograph also, the occurrence of abnormal chest Xray decreased from about $70 \%$ to $20 \%(\mathrm{p}<0.001)$. HRCT thorax (Figures 1 and 2) was performed in all the subjects both at the time of presentation and follow up. At the time of first visit, none of the patient had a normal scan while it was normal in about $45 \%(n=22)$ patients at post-treatment follow up. Most common finding observed at presentation was diffuse ground glass opacities $(n=24 ; 48.97 \%)$ followed by diffuse reticulations $(\mathrm{n}=18 ; 36.73 \%)$ which remained in 7 (14.28\%) and 11 (22.44) patients respectively on follow up.

Further we compared the clinico-radiological findings pre and post treatment in patients given prolonged oral corticosteroids $(\mathrm{n}=24)$ and these are depicted in Table 3. Out of these, nearly 58\% $(\mathrm{n}=14)$ patients had modified Medical Research Council (MMRC) grade 4 breathlessness, which decreased to grade 2 MMRC in about $86 \%(n=12)$ of these patients. Also, the median MMRC score showed significant decrease (Table 3). Majority of patients who were tachypnoeic and hypoxic at rest $(\mathrm{n}=7)$ also showed improvement $(71 \%, n=5)$ post-treatment with corticosteroids. Similarly, patients showed improvement on exertional desaturation also and the average distance covered in 6MWT increased from 291.4 \pm 149.2 meters to $367.3 \pm 109.7$ meters with a mean gain of 75.9 meters.

\section{Discussion}

COVID-19 has affected more than 150 million individuals worldwide [1]. Even after more than 4 weeks have elapsed from
Table 1. Baseline characteristics of 'long-COVID' patients $(n=49)$.

\begin{tabular}{lc}
\hline Gender - Male:female & $34: 15$ \\
Age (mean \pm SD) (in years) & $48.75 \pm 16.56$ \\
\hline Course of COVID-19 & $\mathrm{n}(\%)$ \\
i. Home isolation & $15(30.61)$ \\
ii. Admission without oxygen support & $7(14.28)$ \\
iii. Admission with oxygen support & $15(30.61)$ \\
iv. Non-invasive ventilation (NIV) & $7(14.28)$ \\
v. Invasive mechanical ventilation (IMV) & $5(10.20)$ \\
Pre-existing comorbidities & \\
i. No comorbidities & $11(22.44)$ \\
ii. Non respiratory comorbidities & $24(48.97)$ \\
a. Hypertension & $7(14.28)$ \\
b. Diabetes mellitus & $17(34.69)$ \\
d. Coronary artery disease and dyslipidaemia & $1(2.04)$ \\
d. Hypothyroidism & $4(8.16)$ \\
iii. Respiratory comorbidities & $17(34.69)$ \\
a. Allergic rhinitis & $9(18.36)$ \\
b. Bronchial asthma & $3(6.12)$ \\
c. Bronchial asthma with allergic rhinitis & $1(2.85)$ \\
d. Chronic obstructive pulmonary disease (COPD) & $2(5.71)$ \\
e. Interstitial lung disease (ILD) & $2(5.71)$ \\
\hline Treatment given for long-COVID symptoms & \\
i. Domiciliary oxygen therapy & $7(14.28)$ \\
ii. Prolonged and tapering doses of oral corticosteroids & $24(48.97)$ \\
a. Without any respiratory comorbidity & $18(36.73)$ \\
b. With respiratory comorbidity & $6(12.24)$ \\
iii. Inhaled corticosteroids + long-acting beta agonist & $20(40.81)$ \\
a. Without any respiratory comorbidity & $12(24.49)$ \\
b. With respiratory comorbidity & $8(16.32)$ \\
iv. Oral + inhaled corticosteroids both & $7(14.28)$ \\
v. Short course (7 days) of oral corticosteroids & $8(16.32)$ \\
vi. Oral acebrophylline & $41(83.67)$ \\
\hline
\end{tabular}

Table 2. Follow up of 'long-COVID' patients ( $n=49)$.

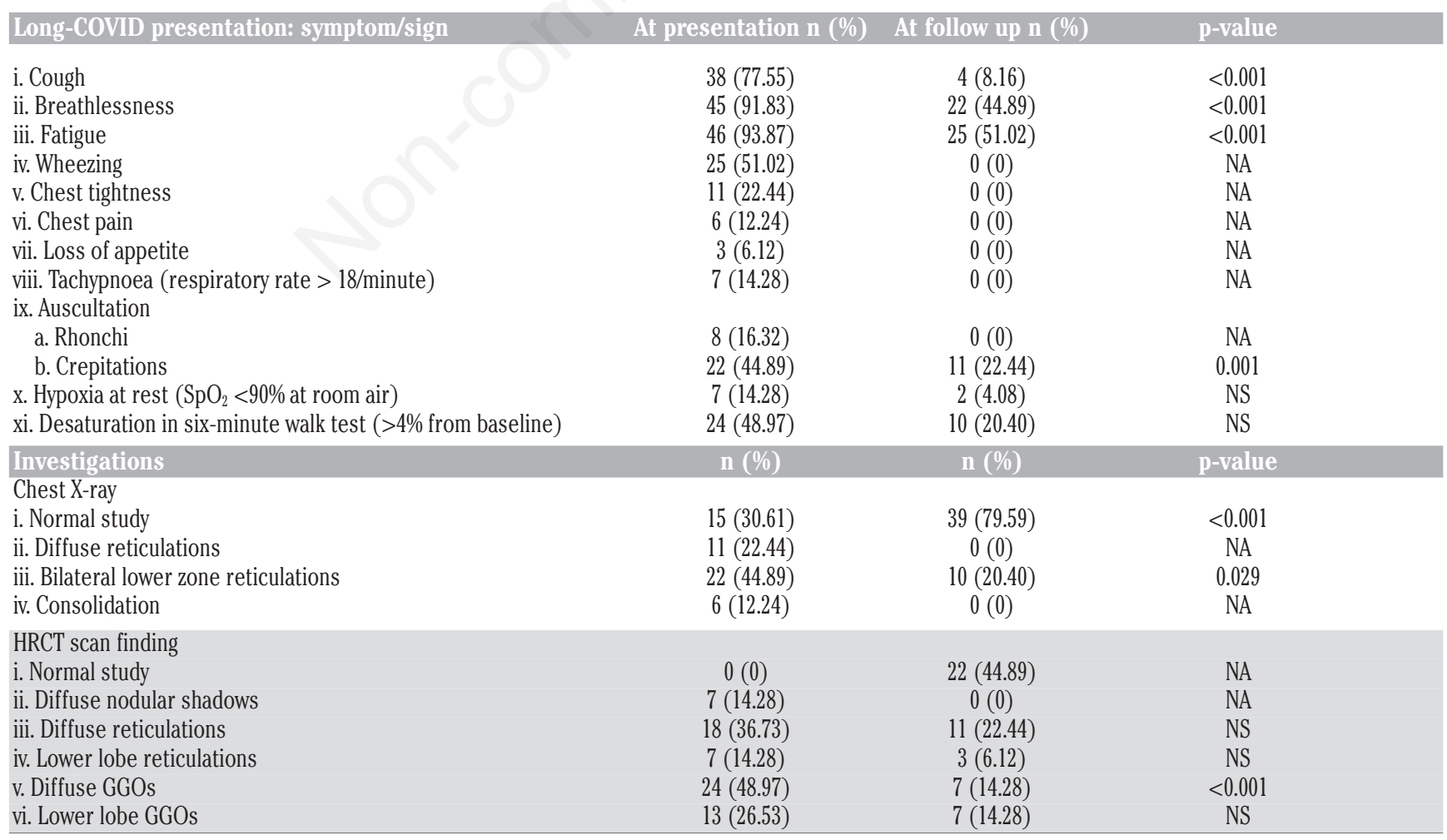

$\mathrm{NA}$, statistical test could not be performed as the patients belong to one category; NS, not significant; GGO, ground glass opacities. 
SARS-CoV-2 positivity, many patients continue to experience persistent symptoms. These symptoms can vary from mild to severe due to extensive lung damage [6]. Also, COVID-19 being a novel disease, not much is known about its long-term implications on the human body. Like another coronavirus i.e. SARS (severe acute respiratory syndrome) virus, COVID-19 is also expected to cause long term residual effects [15]. These residual long-term effects of COVID-19 when persisting after more than 4 weeks from acute infection are designated as 'long-COVID' [9]. Despite some guidance $[9,16]$ now available on the subject, discrete guidelines regarding the pharmacological management of these long-COVID patients are still lacking. In the present study, we assessed clinical symptoms and radiological profile of the 49 long-COVID patients. Further we retrospectively evaluated the effect of treatment given to these patients at about 12 weeks.

Some patients presented to us with history of only mild symp- toms or no symptoms at all during the COVID positivity period. Despite this these patients were symptomatic and in the postCOVID also showed radiological evidence of lung damage. Many studies have studied follow up and analysed the course of hospitalised COVID-19 patients. In a study from France by Garrigues et $a l$., it was found that about 3 months after discharge from the hospital, almost $42 \%$ of patients had persistent dyspnoea [10]. Similarly, a study conducted in China, followed COVID-19 patients every 3 monthly for one year post discharge and found that almost $24 \%$ had persistent radiological abnormalities even after 12 months of acute illness [12]. In a previous study on evaluation of post-COVID patients, it was found that almost $48 \%$ of symptomatic patients had suffered mild COVID-19 with history of home isolation only [17]. In the present study also, we have found that almost $30 \%$ of the patients had history of mild COVID-19 only.

In the present study, more than $75 \%$ long-COVID patients had

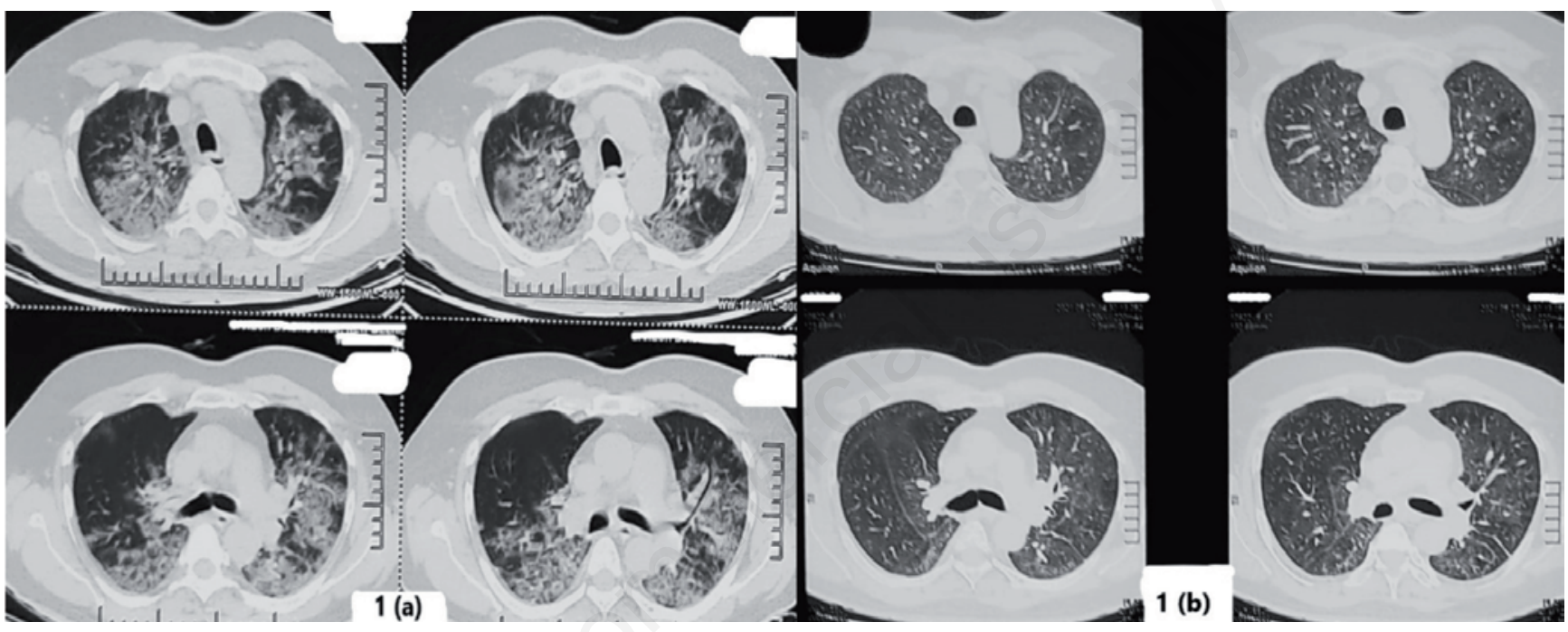

Figure 1. a) CT scan of the chest of a previously healthy male after COVID 19 infection showing bilateral diffuse patches of consolidation. b) CT scan after 3 months of treatment showing notable improvement bilaterally.
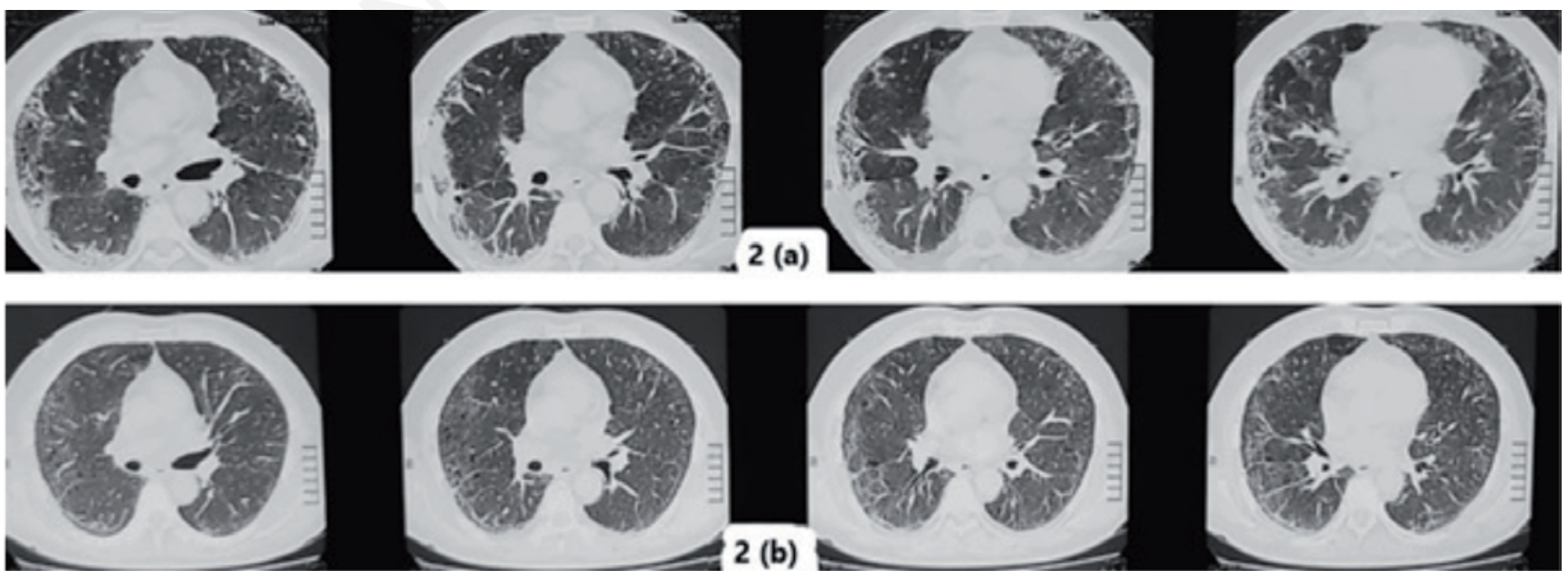

Figure 2. a) CT scan of the chest of a known case of COPD showed bilateral subpleural ground glass opacities (GGOs) after COVID19 infection. b) CT scan after 3 months of treatment showing clearing of bilateral GGOs. 
one or more comorbidities. Further people with comorbidities like diabetes mellitus, hypertension etc have a worse course of acute COVID-19 illness and hence have a higher predisposition to develop long-COVID $[18,19]$. Diabetes mellitus was the most prevalent comorbidity $(\mathrm{n}=17 ; 34.69 \%)$ in the study subjects. Studies have also found that diabetics with poor glycaemic control are more prone to develop lung fibrosis and even death [20,21]. In the current study, all patients with diabetes mellitus as comorbidity had abnormal chest radiography at presentation. Moreover, it has been suggested that COVID-19 disease can result in new diagnosis of diabetes mellitus as well [22]. Our study also showed similar results, where nearly $8 \%$ patients were detected with raised blood sugars for the first time during COVID-19.

Guidelines on 'long-COVID' formulated till now, have not given clear roadmap of pharmacological management for e.g., systemic corticosteroids use, in these patients [9]. Although some guidelines have given suggestion for the use of systemic corticosteroids in this scenario [16]. Further systemic corticosteroids are the cornerstone of treatment in interstitial lung diseases (ILD) presenting with acute respiratory failure (with a few exceptions like vasculitis associated ILD's) [13]. In a corollary to this, we gave long-COVID patients with hypoxic respiratory failure or exertional desaturation, a trial of corticosteroids in tapering doses titrated to the clinico-radiological response in addition to domiciliary oxygen therapy as indicated.

Almost 3 months after treatment, patients showed statistically significant improvement in fatigue, breathlessness and cough. All other signs and symptoms like wheezing, chest tightness, chest pain and tachypnoea also showed resolution (Table 2). Six-minute walk distance increased in most of the subjects with an average gain of 75 meters. Post-treatment at about 3 months, occurrence of resting hypoxia and exertional desaturation showed decrease (from $14 \%$ to $4 \%$ and $49 \%$ to $20 \%$, respectively). Although previous studies have found significant improvement in grades of dyspnoea and gain in six-minute walk distance in post-COVID patients at the end of one year follow up even without any treatment (no mention of any specific treatment) [12]. In a study by Myall et al. [23], the utility of steroids in treatment of patients with persistent inflammatory ILD post-coronavirus infection was studied. They found that the use of systemic steroids in post-COVID-19 ILD, led to significant improvement in dyspnoea, 6MWD, pulmonary function parameters as well as radiology. Another small study [24], also found benefit of systemic steroids on clinical, functional and radiological parameters in patients with long-Covid-ILD.

Earlier studies of follow up of severe COVID-19 patients have demonstrated radiological improvement at 12 weeks in about $22 \%$ [12] and at 6 months in 38\% [25], without any mention of any specific treatment. In the present study, HRCT thorax of all patients had abnormalities on presentation and post treatment about $45 \%$ had normal HRCT (Table 2). Diffuse GGO's showed significant improvement and other HRCT findings also showed resolution. Again, this resolution can be spontaneous or effect of the treatment given or both. Also, the present study comprised a mixed population of COVID-19, with about 30\% having only mild COVID-19.

Despite this, the patients of long-COVID presenting with hypoxemic respiratory failure or exertional desaturation along with high grades of dyspnoea, require management to provide symptomatic relief. We found systemic corticosteroids to be helpful in this situation. In the present study, amongst the pool of patients prescribed oral corticosteroids for post-COVID / long-COVID ( $\mathrm{n}=24$, Table 3 ) about $29 \%(n=7)$ had hypoxia at presentation. Post treatment with corticosteroids, $71.4 \%$ of these patients became normoxic at rest $(\mathrm{p}=0.02)$. Also, the prevalence of exertional desaturation in these patients decreased from $100 \%$ to $41.6 \%$, post-treatment with corticosteroids. Median MMRC score improved from 3 $\left(\mathrm{Q}_{1} 2, \mathrm{Q}_{3} 4\right)$ to $1\left(\mathrm{Q}_{1} 0, \mathrm{Q}_{3} 2\right)(\mathrm{p}<0.001)$. Even the occurrence of abnormal chest X-ray decreased from $87.5 \%$ to $30 \%(\mathrm{p}<0.01)$. Similar to our study, Myall et al. [23] also concluded that early

Table 3. Follow up of 'long-COVID' patients who received prolonged oral corticosteroids $(\mathbf{n}=24)$.

\begin{tabular}{|c|c|c|c|}
\hline Long-COVID presentation: symptom/sign & At presentation n (\%) & At follow upn (\%) & p-value \\
\hline $\begin{array}{l}\text { i. Cough } \\
\text { ii. Breathlessness } \\
\text { iii. Fatigue } \\
\text { iv. Tachypnoea (respiratory rate }>18 / \mathrm{min}) \\
\text { v. Auscultation } \\
\quad \text { a. Rhonchi } \\
\text { b. Crepitations } \\
\text { vi. Hypoxia at rest }\left(\mathrm{SpO}_{2}<90 \% \text { at room air) }\right. \\
\text { vii. Desaturation in } 6 \text {-minute walk test ( }>4 \% \text { from baseline) } \\
\left.\text { viii. MMRC grade [median }\left(\mathrm{Q}_{1}, \mathrm{Q}_{3}\right)\right]^{*}\end{array}$ & $\begin{array}{l}20(83.34) \\
24(100) \\
23(95.84) \\
7(29.17) \\
\\
0(0) \\
17(70.84) \\
7(29.17) \\
24(100) \\
3(2,4)\end{array}$ & $\begin{array}{c}3(12.5) \\
16(66.67) \\
9(37.5) \\
0(0) \\
0(0) \\
9(37.5) \\
2(8.34) \\
10(41.67) \\
1(0,2)\end{array}$ & $\begin{array}{l}<0.001 \\
\text { NA } \\
<0.001 \\
\text { NA } \\
\text { NA } \\
0.004 \\
0.02 \\
\text { NA } \\
<0.001\end{array}$ \\
\hline Investigations & n $(\%)$ & n (\%) & p-value \\
\hline $\begin{array}{l}\text { Chest X-ray } \\
\text { i. Normal study } \\
\text { ii. Diffuse reticulations } \\
\text { iii. Bilateral lower zone reticulations } \\
\text { iv. Consolidation }\end{array}$ & $\begin{array}{c}3(12.5) \\
6(25) \\
14(58.34) \\
4(16.67)\end{array}$ & $\begin{array}{c}17(70.84) \\
0(0) \\
7(29.17) \\
0(0)\end{array}$ & $\begin{array}{c}<0.001 \\
\text { NA } \\
\text { NS } \\
\text { NA }\end{array}$ \\
\hline $\begin{array}{l}\text { HRCT scan finding } \\
\text { i. Normal study } \\
\text { ii. Diffuse nodular shadows } \\
\text { iii. Diffuse reticulations } \\
\text { iv. Lower lobe reticulations } \\
\text { v. Diffuse GGOs } \\
\text { vi. Lower lobe GGOs }\end{array}$ & $\begin{array}{c}0(0) \\
3(12.5) \\
13(54.16) \\
2(8.34) \\
18(75) \\
4(16.67)\end{array}$ & $\begin{array}{c}6(25) \\
0(0) \\
8(33.34) \\
1(4.17) \\
7(29.17) \\
4(16.67)\end{array}$ & $\begin{array}{l}\text { NA } \\
\text { NA } \\
\text { NS } \\
\text { NS } \\
0.013 \\
\text { NS }\end{array}$ \\
\hline
\end{tabular}

NA, statistical test could not be performed as the patients belong to one category; NS, not significant; MMRC, Modified Medical Research council; GGO, ground glass opacities; *Wilcoxon Signed Rank test was performed. 
treatment with corticosteroids resulted in significant improvement in patients with persistent post-COVID-ILD.

Although question remains whether these changes self-resolve or subside as found by other studies [12,25], we understand that above subset of patients warrant therapeutic intervention to provide quicker relief. Also, we cannot overemphasize the caution against the immunosuppressive effects of systemic corticosteroids. Hence, systemic corticosteroids ought to be given only after ruling out infection and simultaneously, they should be rapidly tapered off in relation to the clinical response.

Current study had few limitations as well. Firstly, it was not a planned study and only a retrospective analysis of the management of a small number of long-COVID patients. Also, we did not have a control group for better interpretation of results. Secondly, we could not perform pulmonary function test (PFT) and functional assessment of the patients was done with the help of six-minute walk test only. This was because of PFT were not being conducted due to the COVID-19 situation, at that time. However, we performed 6MWT as a surrogate for the same. Lastly, patients were evaluated at about 12 weeks of follow up, but we might have found still better resolution with an extended follow up.

\section{Conclusions}

We found systemic corticosteroids lead to clinical and radiological improvement in selected subset of patients with 'longCOVID', namely those with radiological changes associated with hypoxia at rest or exertional desaturation. Simultaneously, we should be watchful for the immunosuppressive effect of systemic corticosteroids which can lead to infections like tuberculosis (especially in endemic Asian countries) and invasive fungal diseases. Hence, corticosteroids should be tapered off as soon as possible on achieving clinical stability. Further large-scale studies with longer follow up durations to enable formulation of standardised treatment plans for such long-COVID patients, are the need of the hour.

\section{References}

1. World Health Organization. Coronavirus Disease (COVID-19) Dashboard. Last accessed: 14th May 2021. Available from: https://covid19.who.int

2. Huang G, Gong T, Wang G, et al. Timely diagnosis and treatment shortens the time to resolution of coronavirus disease (covid-19) pneumonia and lowers the highest and last CT scores from sequential chest CT. AJR Am J Roentgenol 2020;215:367-73.

3. Gandhi RT, Lynch JB, del Rio C. Mild or moderate Covid-19. N Engl J Med 2020;383:1757-66.

4. Huang C, Wang Y, Li X, et al. Clinical features of patients infected with 2019 novel coronavirus in Wuhan, China. Lancet 2020;395:497-506.

5. BMJ Best Practice. Coronavirus disease 2019 (COVID-19) symptoms, diagnosis and treatment. Last accessed: 13th May 2021. Available from: https://bestpractice.bmj.com/topics/enus $/ 3000168$

6. Huang C, Huang L, Wang Y, et al. 6-month consequences of COVID-19 in patients discharged from hospital: a cohort study. Lancet 2021;397:220-32.

7. Abdallah SJ, Voduc N, Corrales-Medina VF, et al. Symptoms, pulmonary function and functional capacity four months after covid-19. Ann Am Thor Soc 2021;18:1912-7.

8. Infectious Diseases Society of America. Post COVID conditions (e.g. long COVID). 2021. Last accessed: 8th May 2021. Available from: https:/www.idsociety.org/covid-19-real-timelearning-network/disease-manifestations--complications/postcovid-syndrome/

9. National Institute for Health and Care Excellence. COVID-19 rapid guideline: managing the long-term effects of COVID-19. NICE guideline [NG188]. 2020. Last accessed: 4th May 2021. Available from: https://www.ncbi.nlm.nih.gov/books/ NBK567263/

10. Garrigues E, Janvier P, Kherabi Y, et al. Post-discharge persistent symptoms and health-related quality of life after hospitalization for COVID-19. J Infect 2020;81:e4-6.

11. Carfì A, Bernabei R, Landi F, Gemelli Against COVID-19 Post-Acute Care Study Group. Persistent symptoms in patients after acute COVID-19. JAMA 2020;324:603-5.

12. Wu X, Liu X, Zhou Y, et al. 3-month, 6-month, 9-month, and 12-month respiratory outcomes in patients following COVID19-related hospitalisation: a prospective study. Lancet Respir Med 2021;9:747-54.

13. Bradley B, Branley HM, Egan JJ, et al. Interstitial lung disease guideline: the British Thoracic Society in collaboration with the Thoracic Society of Australia and New Zealand and the Irish Thoracic Society. Thorax 2008;63:v1-58.

14. Antonisamy A, Premkumar P, Christopher S. Principles and practice of biostatistics. Elsevier India; 2017.

15. Zhang P, Li J, Liu H, et al. Long-term bone and lung consequences associated with hospital-acquired severe acute respiratory syndrome: a 15 -year follow-up from a prospective cohort study. Bone Res 2020;8:8.

16. Kumar R, Behera D, Jindal SK, et al. Post-COVID-19 respiratory management: Expert panel report. Respiratory management. Indian J Chest Dis Allied Sci 2020;62:179-91.

17. Goel N, Goyal N, Kumar R. Clinico-radiological evaluation of post COVID-19 at a tertiary pulmonary care centre in Delhi, India. Monaldi Arch Chest Dis 2021;91:1682.

18. Guan W, Liang W, Zhao Y, et al. Comorbidity and its impact on 1590 patients with COVID-19 in China: a nationwide analysis. Eur Respir J 2020;55:2000547.

19. Goel N, Spalgais S, Mrigpuri P, et al. Characteristics of COVID-19 at a non-COVID tertiary pulmonary care centre in Delhi, India. Monaldi Arch Chest Dis 2020;90:1568.

20. Mrigpuri P, Sonal S, Spalgais S, et al. Uncontrolled diabetes mellitus: A risk factor for post COVID fibrosis. Monaldi Arch Chest Dis 2021;91:1607.

21. Apicella M, Campopiano MC, Mantuano M, et al. COVID-19 in people with diabetes: understanding the reasons for worse outcomes. Lancet Diabetes Endocrinol 2020;8:782-92.

22. Hayden MR. An immediate and long-term complication of COVID-19 may be type 2 diabetes mellitus: The central role of $\beta$-Cell dysfunction, apoptosis and exploration of possible mechanisms. Cells 2020;9:2475.

23. Myall KJ, Mukherjee B, Castanheira AM, et al. Persistent postCOVID-19 interstitial lung disease. An observational study of corticosteroid treatment. Ann Am Thorac Soc 2021;18:799-806.

24. Goel N, Goyal N, Kumar R. Insights in the management of long COVID-19: preliminary observations. Indian J Chest Dis Allied Sci 2021;63:65-73.

25. Han X, Fan Y, Alwalid O, et al. Six-month follow-up chest CT findings after severe COVID-19 pneumonia. Radiology 2021;299:E177-86. 